\title{
ON A GENERALIZATION OF NC-MCCOY RINGS
}

\author{
MOHAMMAD VAHDANI MEHRABADI, SHERVIN SAHEBI, \\ AND HAMID H. S. JAVADI
}

Received 04 November, 2015

\begin{abstract}
In the present paper we concentrate on a natural generalization of NC-McCoy rings that is called J-McCoy and investigate their properties. We prove that local rings are J-McCoy. For a ring $R, R[[x]]$ is $\mathrm{J}-M c C o y$ if and only if $R$ is $\mathrm{J}$-McCoy. Also, for an abelian ring $R$, we show that $R$ is J-McCoy if and only if $e R$ is J-McCoy, where $e$ is an idempotent element of $R$. Moreover, we give an example to show that the J-McCoy property does not pass $M_{n}(R)$, but $S(R, n), A(R, n), B(R, n)$ and $T(R, n)$ are J-McCoy.
\end{abstract}

2010 Mathematics Subject Classification: 34B10; 34B15

Keywords: McCoy ring, weak McCoy ring, J-McCoy ring, polynomial ring, upper triangular matrix ring

\section{INTRODUCTION}

Throughout this paper, $R$ denotes an associative ring with identity. For a ring $R$, $N(R), M_{n}(R)$ and $e_{i j}$ denote the set of all nilpotent elements in $R$, the $n \times n$ matrix ring over $R$, and the matrix with $(i, j)$-entry 1 and elsewhere 0 , respectively. Rege -Chhawchharia [8] called a noncommutative ring $R$ right McCoy if whenever polynomials $f(x)=\sum_{i=0}^{n} a_{i} x^{i}, g(x)=\sum_{j=0}^{m} b_{j} x^{j} \in R[x] \backslash\{0\}$ satisfy $f(x) g(x)=0$, there exists a nonzero element $r \in R$ such that $a_{i} r=0$. Left McCoy rings are defined similarly. A number of papers have been written on McCoy property of rings (see, e.g., $[1,4,6,7,9])$. The name "McCoy" was chosen because McCoy [6] had noted that every commutative ring satisfies this condition. Victor Camillo, Tai Keun Kwak, and Yang Lee [2] called a ring $R$ right nilpotent coefficient McCoy(simply, right NC-McCoy) if whenever polynomials $f(x)=\sum_{i=0}^{n} a_{i} x^{i}, g(x)=\sum_{j=0}^{m} b_{j} x^{j} \in$ $R[x] \backslash\{0\}$ satisfy $f(x) g(x)=0$, there exists a nonzero element $r \in R$ such that $f(x) r \in N(R)[x]$. Left NC-McCoy rings are defined analogously, and a ring $R$ is called NC-McCoy if it is both left and right NC-McCoy. They proved for a reduced

This paper is supported by Islamic Azad University Central Tehran Branch (IAUCTB). The authors want to thank the authority of IAUCTB for their support to complete this research. 
ring $R$ and $n \geq 2, M_{n}(R)$ is neither right nor left NC-McCoy, but $T_{n}(R)$ is a NCMcCOy ring for $n \geq 2$. Moreover, it is shown that $R$ is right NC-McCoy if the polynomial ring $R[x]$ is right NC-McCoy and the converse holds if $N(R)[x] \subset N(R[x])$.

Motivated by the above results, we investigate a generalization of the right NCMcCoy rings. The Jacobson radical is an important tool for studying the structure of noncommutative rings, and denoted by $J(R)$. A ring $R$ is said to be right $\mathrm{J}$ McCoy (respectively left J-McCoy) if for each pair of nonzero polynomials $f(x)=$ $\sum_{i=0}^{n} a_{i} x^{i}$ and $g(x)=\sum_{j=0}^{m} b_{j} x^{j} \in R[x] \backslash\{0\}$ with $f(x) g(x)=0$, then there exists a nonzero element $r \in R$ such that $a_{i} r \in J(R)$ (respectively $r b_{j} \in J(R)$ ). A ring $R$ is called J-McCoy if it is both left and right J-McCoy. It is clear that NC-McCoy rings are $\mathrm{J}$-McCoy, but the converse is not always true. If $\mathrm{R}$ is $\mathrm{J}$-semisimple (namely, $J(R)=0$ ), then $R$ is right $\mathrm{J}-\mathrm{McC}$ cy if and only if $R$ is right McCoy. Moreover, for Artinian rings, the concepts of NC-McCoy and J-McCoy rings are the same.

\section{Results}

Definition 1. A ring $R$ is said to be right J-McCoy (respectively left J-McCoy) if for each pair of nonzero polynomials $f(x)=\sum_{i=0}^{n} a_{i} x^{i}$ and $g(x)=\sum_{j=0}^{m} b_{j} x^{j} \in$ $R[x] \backslash\{0\}, f(x) g(x)=0$ implies that there exists a nonzero element $r \in R$ with $a_{i} r \in J(R)$ (respectively $r b_{j} \in J(R)$ ). A ring $R$ is called J-McCoy if it is both left and right $\mathrm{J}-\mathrm{McCoy}$.

It is clear that NC-McCoy rings are J-McCoy, but the converse is not always true by the following example.

Example 1. Let $A$ be the 3 by 3 full matrix ring over the power series ring $F[[t]]$ over a field $F$. Let

$B=\left\{M=\left(m_{i j}\right) \in A \mid m_{i j} \in t F[[t]]\right.$ for $1 \leq i, j \leq 2$ and $m_{i j}=0$ for $i=3$ or $\left.j=3\right\}$

and

$$
C=\left\{M=\left(m_{i j}\right) \in A \mid m_{i j} \in F \text { and } m_{i j}=0 \text { for } i \neq j\right\} .
$$

Let $R$ be the subring of $A$ generated by $B$ and $C$. Let $F=\mathbb{Z}_{2}$. Note that every element of $\mathrm{R}$ is of the form $\left(a+f_{1}\right) e_{11}+f_{2} e_{12}+f_{3} e_{21}+\left(a+f_{4}\right) e_{22}+a e_{33}$ for some $a \in F$ and $f_{i} \in t F[[t]](i=1,2,3,4)$. Consider two polynomials over $\mathrm{R}, f(x)=$ $t e_{11}+t e_{12} x+t e_{21} x^{2}+t e_{22} x^{3}$ and $g(x)=-t\left(e_{21}+e_{22}\right)+t\left(e_{11}+e_{12}\right) x \in R[x]$. Then $f(x) g(x)=0$. If there exists $0 \neq r \in R$ such that $f(x) r \in N(R[x])$, then $r=0$. Thus $R$ is not right NC-McCoy.

Next we will show that $R$ is right J-McCoy. Let $f(x)=\sum_{i=0}^{n} M_{i} x^{i}$ and $g(x)=$ $\sum_{j=0}^{m} N_{j} x^{j}$ be nonzero polynomials in $R[x]$ such that $f(x) g(x)=0$. Since $M_{i}=$ $\left(a_{i}+f_{i 1}\right) e_{11}+f_{i 2} e_{12}+f_{i 3} e_{21}+\left(a_{i}+f_{i 4}\right) e_{22}+a_{i} e_{33}$ for some $a_{i} \in F$ and $f_{i j} \in$ $t F[[t]](j=0,1,2,3,4)$, then for $C=t e_{11}$ we have $M_{i} C=\left(a_{i}+f_{i 1}\right) t e_{11}+f_{i 3} t e_{21}$ $\in J(R)$. Thus $R$ is right $\mathrm{J}-\mathrm{McCoy}$ ring. 
Proposition 1. Let $R$ be a ring and $I$ an ideal of $R$ such that $R / I$ is a right (resp. left) $J$-McCoy ring. If $I \subseteq J(R)$, then $R$ is a right (resp. left) $J$-McCoy ring.

Proof. Suppose that $f(x)=\sum_{i=0}^{m} a_{i} x^{i}$ and $g(x)=\sum_{j=0}^{n} b_{j} x^{j} \in R[x] \backslash\{0\}$ such that $f(x) g(x)=0$. Then $\left(\sum_{i=0}^{m} \overline{a_{i}} x^{i}\right)\left(\sum_{j=0}^{n} \overline{b_{j}} x^{j}\right)=\overline{0}$ in $R / I$. Thus there exists $\bar{c} \in R / I$ such that $\overline{a_{i}} \bar{c} \in J(R / I)$ and so $a_{i} c \in J(R)$. This means $R$ is right J-McCoy ring.

Corollary 1. Let $R$ be any local ring. Then $R$ is J-McCoy.

The following example shows that, if $R$ is a right J-McCoy ring, then $R / J(R)$ is not necessary right J-McCoy.

Example 2. Let $R$ denote the localization of the ring $\mathbb{Z}$ of integers at the prime ideal (3). Consider the quaternions $\mathbb{Q}$ over $R$, that is, a free R-module with basis $1, i, j, k$ and multiplication satisfying $i^{2}=j^{2}=k^{2}=-1, i j=k=-j i$. Then $\mathbb{Q}$ is a noncommutative domain, and so $J(\mathbb{Q})=3 \mathbb{Q}$ and $\mathbb{Q} / J(\mathbb{Q})$ is isomorphic to the 2-by-2 full matrix ring over $\mathbb{Z} /(3)$. Thus $\mathbb{Q}$ is a right J-McCoy ring, but $\mathbb{Q} / J(\mathbb{Q})$ is not right J-McCoy.

Proposition 2. Let $R_{k}$ be a ring, where $k \in I$. Then $R_{k}$ is right (resp. left) $J$-McCoy for each $k \in I$ if and only if $R=\prod_{k \in I} R_{k}$ is right (resp. left) J-McCoy.

Proof. Let each $R_{k}$ be a right J-McCoy ring and $f(x)=\sum_{i=0}^{m} a_{i} x^{i}, g(x)=$ $\sum_{j=0}^{n} b_{j} x^{j} \in R[x] \backslash\{0\}$ such that $f(x) g(x)=0$, where $a_{i}=\left(a_{i}{ }^{(k)}\right), b_{j}=\left(b_{j}{ }^{(k)}\right)$. If there exists $t \in I$ such that $a_{i}^{(t)}=0$ for each $0 \leq i \leq m$, then we have $a_{i} c=0 \in J(R)$ where $c=\left(0,0, \ldots, 1_{R_{t}}, 0, \ldots, 0\right)$. Now suppose for each $k \in I$, there exists $0 \leq i_{k} \leq$ $m$ such that $a_{i_{k}}^{(k)} \neq 0$. Since $g(x) \neq 0$, there exists $t \in I$ and $0 \leq j_{t} \leq n$ such that $b_{j_{t}}^{(t)} \neq 0$. Consider $f_{t}(x)=\sum_{i=0}^{m} a_{i}^{(t)} x^{i}$ and $g_{t}(x)=\sum_{i=0}^{n} b_{j}^{(t)} x^{j} \in R_{t}[x] \backslash\{0\}$. We have $f_{t}(x) g_{t}(x)=0$. Thus there exists nonzero $c_{t} \in R_{t}$ such that $a_{i}^{(t)} c_{t} \in J\left(R_{t}\right)$, for each $0 \leq i \leq m$, since $R_{t}$ is right J-McCoy ring. Therefore, $a_{i}\left(0,0, \ldots, c_{t}, 0, \ldots, 0\right) \in$ $\prod_{k \in I} J\left(R_{k}\right)=J(R)$, for each $0 \leq i \leq m$. Thus, $R$ is right $\mathrm{J}-\mathrm{McCoy}$.

Conversely, suppose $R$ is right J-McCoy and $t \in I$. Let $f(x)=\sum_{i=0}^{m} a_{i} x^{i}, g(x)=$ $\sum_{j=0}^{n} b_{j} x^{j}$ be nonzero polynomials in $R_{t}[x]$ such that $f(x) g(x)=0$. Set

$$
\begin{aligned}
& F(x)=\sum_{i=0}^{m}\left(0,0, \ldots, 0, a_{i}, 0, \ldots, 0\right) x^{i}, \\
& G(x)=\sum_{j=0}^{n}\left(0,0, \ldots, 0, b_{j}, 0, \ldots, 0\right) x^{j} \in R[x] \backslash\{0\} .
\end{aligned}
$$

Hence $F(x) G(x)=0$ and so there exists $0 \neq c=\left(c_{i}\right)$ such that $\left(0,0, \ldots, 0, a_{i}, 0, \ldots, 0\right) c \in J(R)=\prod_{k \in I} J\left(R_{k}\right)$. Therefore, $a_{i} c_{t} \in J\left(R_{t}\right)$ and so $R_{t}$ is right $\mathrm{J}-\mathrm{McCoy}$ 
Corollary 2. Let $D$ be a ring and $C$ a subring of $D$ with $1_{D} \in C$. Let

$$
R(C, D)=\left\{\left(d_{1}, \ldots, d_{n}, c, c, \ldots\right) \mid d_{i} \in D, c \in C, n \geq 1\right\}
$$

with addition and multiplication defined component-wise, $R(D, C)$ is a ring. Then $D$ is right (resp. left) $J-M c C o y$ if and only if $R(D, C)$ is right (resp. left) J-McCoy.

Theorem 1. The class of right (resp. left) J-McCoy rings is closed under direct limits with injective maps.

Proof. Let $D=\left\{R_{i}, \alpha_{i j}\right\}$ be direct system of right J-McCoy rings $R_{i}$, for $i \in I$ and ring homomorphisms $\alpha_{i j}: R_{i} \longrightarrow R_{j}$ for each $i \leq j$ satisfying $\alpha_{i j}(1)=1$, where $I$ is a directed partially ordered set. Set $R=\lim R_{i}$ be a direct limit of $D$ with

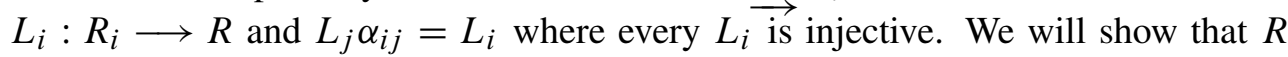
is an right J-McCoy ring. Take $a, b \in R$. Then $a=L_{i}\left(a_{i}\right), b=L_{j}\left(b_{j}\right)$ for some $i, j \in I$ and there is $k \in I$ such that $i \leq k, j \leq k$. Define

$$
a+b=L_{k}\left(\alpha_{i k}\left(a_{i}\right)+\alpha_{j k}\left(b_{j}\right)\right) \text { and } a b=L_{k}\left(\alpha_{i k}\left(a_{i}\right) \alpha_{j k}\left(b_{j}\right)\right)
$$

where $\alpha_{i k}\left(a_{i}\right)$ and $\alpha_{j k}\left(b_{j}\right)$ are in $R_{k}$. Then $R$ forms a ring with $0=L_{i}(0)$ and $1=L_{i}(1)$. Now let $f(x)=\sum_{i=0}^{n} a_{i} x^{i}$ and $g(x)=\sum_{j=0}^{m} b_{j} x^{j} \in R[x]$ be nonzero polynomials such that $f(x) g(x)=0$. There is $k \in I$ such that $f(x), g(x) \in R_{k}[x]$. Hence we get $f(x) g(x)=0$ in $R_{k}[x]$. Since $R_{k}$ is right J-McCoy, there exist $0 \neq c_{k}$ in $R_{k}$ such that $a_{i} c_{k} \in J\left(R_{k}\right)$. Put $c=L_{k}\left(c_{k}\right)$. Then $a_{i} c \in \underset{\lim }{\longrightarrow} J\left(R_{k}\right)=J(R)$ with a nonzero $c$ in $R$. Thus $R$ is right $\mathrm{J}$-McCoy ring.

Theorem 2. For a ring $R, R[[x]]$ is right (resp. left) $J$-McCoy if and only if $R$ is right (resp. left) J-McCoy.

Proof. Let $R$ be a right J-McCoy ring. Since $R \cong \frac{R[[x]]}{<x>}$ and $<x>\subset J(R[[x]])$, then by Proposition $1, R[[x]]$ is right J-McCoy. Conversely, assume that $R[[x]]$ is right J-McCoy. Let $f(y)=\sum_{i=0}^{n} a_{i} y^{i}$ and $g(y)=\sum_{j=0}^{m} b_{j} y^{j}$ be nonzero plynomials $\in R[y]$, such that $f(y) g(y)=0$. Since $R[[x]]$ is right J-McCoy and $R \subseteq$ $R[[x]]$, then there exists $0 \neq c(x)=c_{0}+c_{1} x+c_{2} x+\ldots \in R[[x]]$ such that $a_{i} c(x) \in$ $J(R[[x]])$ and so $a_{i} c_{i} \in J(R[[x]]) \cap R \subseteq J(R)$ for all $i=0,1, \cdots, n$. Since $c(x)$ is nonzero, there exists $c_{l} \neq 0$ such that $a_{i} c_{l} \in J(R)$ for $i=0,1, \cdots, n$ and so $R$ is $J$-McCoy.

Theorem 3. For a ring $R$, if $R[x]$ is right (resp. left) $J-M c C o y$, then $R$ is right (resp. left) J-McCoy. The converse holds if $J(R)[x] \subseteq J(R[x])$.

Proof. Suppose that $R[x]$ is right J-McCoy. Let $f(y)=\sum_{i=0}^{n} a_{i} y^{i}$ and $g(y)=$ $\sum_{j=0}^{m} b_{j} y^{j}$ be nonzero plynomials in $R[y]$, such that $f(y) g(y)=0$. Since $R[x]$ is right J-McCoy and $R \subseteq R[x]$, then there exists $0 \neq c(x)=c_{0}+c_{1} x+\ldots+c_{k} x^{k} \in$ $R[x]$ such that $a_{i} c(x) \in J(R[x])$ and so $a_{i} c_{i} \in J(R[x]) \cap R \subseteq J(R)$ for all $i=$ $0,1, \cdots, n$. Since $c(x)$ is nonzero, there exists $c_{l} \neq 0$ such that $a_{i} c_{l} \in J(R)$ for 
$i=0,1, \cdots, n$ and so $R$ is $\mathrm{J}-\mathrm{McC}$ Cy. Conversely, suppose that $R$ is right J-McCoy and $f(y) g(y)=0$ for nonzero polynomials $f(y)=f_{0}+f_{1} y+\ldots+f_{m} y^{m}$ and $g(y)=g_{0}+g_{1} y+\ldots+g_{n} y^{n}$ in $(R[x])[y]$. Take the positive integer $\mathrm{k}$ with $k=$ $\sum_{i=0}^{m} \operatorname{deg} f_{i}+\sum_{j=0}^{n} \operatorname{deg} g_{j}$ where the degree of the zero polynomial is taken to be zero. Then $f\left(x^{k}\right)$ and $g\left(x^{k}\right)$ are nonzero polynomials in $R[x]$ and $f\left(x^{k}\right) g\left(x^{k}\right)=0$, since the set of coefficients of the $f_{i}$ 's and $g_{j}$ 's coincide with the set of coefficients of $f\left(x^{k}\right)$ and $g\left(x^{k}\right)$. Since $\mathrm{R}$ is right J-McCoy, there exists a nonzero element $c \in R$ such that $a_{i} c \in J(R)$, for any coefficient $a_{i}$ of $f_{i}(x)$. So $f_{i} c \in J(R)[x] \subseteq J(R[x])$. Thus $R[x]$ is right $\mathrm{J}-\mathrm{McCoy}$.

Recall that a ring $R$ is said to be abelian if every idempotent of it is central.

Proposition 3. Let $R$ be a right (resp. left) J-McCoy ring and e be an idempotent element of $R$. Then eRe is a right (resp. left) J-McCoy ring. The converse holds if $R$ is an abelian ring.

Proof. Consider $f(x)=\sum_{i=0}^{n} e a_{i} e x^{i}, g(x)=\sum_{j=0}^{m} e b_{j} e x^{j} \in(e R e)[x] \backslash\{0\}$ such that $f(x) g(x)=0$. Since $R$ is a right J-McCoy ring, there exists $s \in R$ such that $\left(e a_{i} e\right) s \in J(R)$. So $\left(e a_{i} e\right) e s e \in e J(R) e=J(e R e)$. Hence $e R e$ is right J-McCoy. Now, assume that $e R e$ is a right J-McCoy ring. Consider $f(x)=\sum_{i=0}^{n} a_{i} x^{i}, g(x)=$ $\sum_{j=0}^{m} b_{j} x^{j} \in R[x] \backslash\{0\}$ such that $f(x) g(x)=0$. Clearly, ef $(x) e, e g(x) e \in(e R e)[x]$ and $(e f(x) e)(e g(x) e)=0$, since $e$ is a central idempotent element of $R$. Then there exists $s \in e \operatorname{Re}$ such that $\left(e a_{i} e\right) s=\left(a_{i}\right) s \in J(e R e)=e J(R) \subset J(R)$. Hence, $R$ is right J-McCoy.

The following example shows that, if $R$ is a right J-McCoy ring, then $M_{n}(R)$ is not necessary right J-McCoy for $n \geq 2$, i.e. the $J$-McCoy property is not Morita invariant.

Example 3. Let $\mathbb{Z}$ be the set of integers. It's clear that $\mathbb{Z}$ is J-McCoy, but $M_{3}(\mathbb{Z})$ is not right $\mathrm{J}-\mathrm{McCoy}$. For

$$
f(x)=\left(\begin{array}{ccc}
1 & x & x^{2} \\
x^{3} & x^{4} & x^{5} \\
x^{6} & x^{7} & x^{8}
\end{array}\right) \text { and } g(x)=\left(\begin{array}{ccc}
x & x & x \\
-1 & -1 & -1 \\
0 & 0 & 0
\end{array}\right)
$$

in $M_{3}(\mathbb{Z})[x]$, we have $f(x) g(x)=0$. Assume to the contrary that $M_{3}(\mathbb{Z})$ is right $\mathrm{J}$-McCoy, then there exists $c=\left(c_{i j}\right) \in M_{3}(\mathbb{Z})$ such that $\left(E_{i j} c\right) \in J\left(M_{3}(\mathbb{Z})\right)=$ $M_{3}(J(\mathbb{Z}))=0$ for $i, j=1,2,3$. This implies $c=0$, which is a contradiction.

Let $R$ be a ring and $\sigma$ denote an endomorphism of $R$ with $\sigma(1)=1$. In [3] the authors introduced skew triangular matrix ring as a set of all triangular matrices with addition point-wise and a new multiplication subject to the condition $E_{i j} r=\sigma^{j-i} E_{i j}$. So $\left(a_{i j}\right)\left(b_{i j}\right)=\left(c_{i j}\right)$, where $c_{i j}=a_{i j} b_{i j}+a_{i, i+1} \sigma\left(b_{i+1, j}\right)+\ldots+a_{i j} \sigma^{j-i}\left(b_{j j}\right)$, for each $i \leq j$ and denoted it by $T_{n}(R, \sigma)$. The subring of the skew triangular matrices with constant mail diagonal is denoted by $S(R, n, \sigma)$; and the subring of the skew 
triangular matrices with constant diagonals is denoted by $T(R, n, \sigma)$. We can denote $A=\left(a_{i j}\right) \in T(R, n, \sigma)$ by $\left(a_{11}, \ldots, a_{1 n}\right)$. Then $T(R, n, \sigma)$ is a ring with addition point-wise and multiplication given by $\left(a_{0}, \ldots, a_{n-1}\right)\left(b_{0}, \ldots, b_{n-1}\right)=\left(a_{0} b_{0}, a_{0} * b_{1}+\right.$ $\left.a_{1} * b_{0}, \ldots, a_{0} * b_{n-1}+\ldots+a_{n-1} * b_{0}\right)$, with $a_{i} * b_{j}=a_{i} \sigma^{i}\left(b_{j}\right)$, for each $i$ and $j$. Therefore, clearly one can see that $T(R, n, \sigma) \cong R[x ; \sigma] /\left(x^{n}\right)$ is the ideal generated by $x^{n}$ in $R[x ; \sigma]$. we consider the following two subrings of $S(R, n, \sigma)$, as follows (see [3]):

$$
\begin{aligned}
& A(R, n, \sigma)=\sum_{j=1}^{\left[\frac{n}{2}\right]} \sum_{i=1}^{n-j+1} a_{j} E_{i, i+j-1}+\sum_{j=\left[\frac{n}{2}\right]+1}^{n} \sum_{i=1}^{n-j+1} a_{i, i+j-1} E_{i, i+j-1}, \\
& B(R, n, \sigma)=\left\{A+r E_{1 k} \mid A \in A(R, n, \sigma) \text { and } r \in R\right\} \quad n=2 k \geq 4
\end{aligned}
$$

In the special case, when $\sigma=i d_{R}$, we use $S(R, n), A(R, n), B(R, n)$ and $T(R, n)$ instead of $S(R, n, \sigma), A(R, n, \sigma), B(R, n, \sigma)$ and $T(R, n, \sigma)$, respectively.

Proposition 4. Let $R$ be a ring. Then $S$ is right J-McCoy ring, for $n \geq 2$, where $S$ is one of the rings $T_{n}(R, \sigma), S(R, n, \sigma), T(R, n, \sigma), A(R, n, \sigma)$ or $B(R, n, \sigma)$.

Proof. Let $f(x)=A_{0}+A_{1} x+\ldots+A_{p} x^{p}, g(x)=B_{0}+B_{1} x+\ldots+B_{q} x^{q}$ be elements of $S[x]$ satisfying $f(x) g(x)=0$ where the $(1,1)-t h$ entry of $A_{i}$ is $a_{11}^{(i)}$. Then $A_{i} E_{1 n}=a_{11}^{(i)} E_{1 n} \in J(S)$ and the proof is complete.

Let $R$ and $S$ be two rings, and Let $M$ be an $(R, S)$-bimodule. This means that $M$ is a left $R$-module and a right $S$-module such that $(\mathrm{rm}) s=r(\mathrm{~ms})$ for all $r \in R$, $m \in M$, and $s \in S$. Given such a bimodule $M$ we can form

$$
T=\left(\begin{array}{cc}
R & M \\
0 & S
\end{array}\right)=\left\{\left(\begin{array}{cc}
r & m \\
0 & s
\end{array}\right): r \in R, m \in M, s \in S\right\}
$$

and define a multiplication on $T$ by using formal matrix multiplication:

$$
\left(\begin{array}{ll}
r & m \\
0 & s
\end{array}\right)\left(\begin{array}{cc}
r^{\prime} & m^{\prime} \\
0 & s^{\prime}
\end{array}\right)=\left(\begin{array}{ccc}
r r^{\prime} & r m^{\prime}+m s^{\prime} \\
0 & s s^{\prime}
\end{array}\right) \text {. }
$$

This ring construction is called triangular ring $T$.

Proposition 5. Let $R$ and $S$ be two rings and $T$ be the triangular ring $T=\left(\begin{array}{cc}R & M \\ 0 & S\end{array}\right)$ (where $M$ is an $(R, S)$-bimodule). Then the rings $R$ and $S$ are right (resp. left) $J$ $M c$ Coy if and only if $T$ is right (resp. left) J-McCoy.

Proof. Assume that $R$ and $S$ are two right J-McCoy rings. Take $I=\left(\begin{array}{cc}0 & M \\ 0 & 0\end{array}\right)$, then $T / I \simeq\left(\begin{array}{cc}R & 0 \\ 0 & S\end{array}\right)$. Let

$$
\begin{gathered}
f(x)=\left(\begin{array}{cc}
r_{0} & 0 \\
0 & s_{0}
\end{array}\right)+\left(\begin{array}{cc}
r_{1} & 0 \\
0 & s_{1}
\end{array}\right) x+\cdots+\left(\begin{array}{cc}
r_{n} & 0 \\
0 & s_{n}
\end{array}\right) x^{n}, \\
g(x)=\left(\begin{array}{cc}
r_{0}^{\prime} & 0 \\
0 & s_{0}^{\prime}
\end{array}\right)+\left(\begin{array}{cc}
r_{1}^{\prime} & 0 \\
0 & s_{1}^{\prime}
\end{array}\right) x+\cdots+\left(\begin{array}{cc}
r_{m}^{\prime} & 0 \\
0 & s_{m}^{\prime}
\end{array}\right) x^{m} \in T[x]
\end{gathered}
$$

satisfy $f(x) g(x)=0$. Define 


$$
f_{r}(x)=r_{0}+r_{1} x+\cdots+r_{n} x^{n}, g_{r}(x)=r_{0}^{\prime}+r_{1}^{\prime} x+\cdots+r_{m}^{\prime} x^{m} \in R[x]
$$

and

$$
f_{s}(x)=s_{0}+s_{1} x+\cdots+s_{n} x^{n}, g_{s}(x)=s_{0}^{\prime}+s_{1}^{\prime} x+\cdots+s_{m}^{\prime} x^{m} \in S[x] .
$$

From $f(x) g(x)=0$, we have $f_{r}(x) g_{r}(x)=f_{s}(x) g_{s}(x)=0$. Since $R$ and $S$ are right J-McCoy rings, then there exists $c \in R$ and $d \in S$ such that $r_{i} c \in J(R)$ and $s_{i} d \in J(S)$ for any $1 \leq i \leq n$ and $1 \leq j \leq m$. Hence if we put $I=\left(\begin{array}{ll}0 & M \\ 0 & 0\end{array}\right)$ then $T / I$ is right $\mathrm{J}$-McCoy and so $T$ is right $\mathrm{J}$-McCoy by Proposition 1. Conversely, let $T$ be a right J-McCoy ring, $f_{r}(x)=r_{0}+r_{1} x+\cdots+r_{n} x^{n}, g_{r}(x)=r_{0}^{\prime}+r_{1}^{\prime} x+$ $\cdots+r_{m}^{\prime} x^{m} \in R[x]$, such that $f_{r}(x) g_{r}(x)=0$, and $f_{s}(x)=s_{0}+s_{1} x+\cdots+s_{n} x^{n}$, $g_{s}(x)=s_{0}^{\prime}+s_{1}^{\prime} x+\cdots+s_{m}^{\prime} x^{m} \in S[x]$, such that $f_{s}(x) g_{s}(x)=0$. Let

$$
\begin{aligned}
& f(x)=\left(\begin{array}{cc}
r_{0} & 0 \\
0 & s_{0}
\end{array}\right)+\left(\begin{array}{cc}
r_{1} & 0 \\
0 & s_{1}
\end{array}\right) x+\cdots+\left(\begin{array}{cc}
r_{n} & 0 \\
0 & s_{n}
\end{array}\right) x^{n} \text { and } \\
& g(x)=\left(\begin{array}{cc}
r_{0}^{\prime} & 0 \\
0 & s_{0}^{\prime}
\end{array}\right)+\left(\begin{array}{cc}
r_{1}^{\prime} & 0 \\
0 & s_{1}^{\prime}
\end{array}\right) x+\cdots+\left(\begin{array}{cc}
r_{m}^{\prime} & 0 \\
0 & s_{m}^{\prime}
\end{array}\right) x^{m} \in T[x] .
\end{aligned}
$$

Then $f_{r}(x) g_{r}(x)=0$ and $f_{s}(x) g_{s}(x)=0$ implies that $f(x) g(x)=0$. Since $T$ is a right J-McCoy ring then there exists $\left(\begin{array}{cc}c & m \\ 0 & d\end{array}\right) \in T$ such that $\left(\begin{array}{cc}r_{i} & 0 \\ 0 & s_{i}\end{array}\right)\left(\begin{array}{ll}c & m \\ 0 & d\end{array}\right) \in J(T)=$ $\left(\begin{array}{cc}J(R) & M \\ 0 & J(S)\end{array}\right)$. Thus $r_{i} c \in J(R)$ and $s_{i} d \in J(S)$ for any $i, j$. This shows that $R$ and $S$ are right J-McCoy.

Given a ring $R$ and a bimodule ${ }_{R} M_{R}$, the trivial extension of $R$ by $M$ is the ring $T(R, M)=R \oplus M$ with the usual addition and the multiplication

$$
\left(r_{1}, m_{1}\right)\left(r_{2}, m_{2}\right)=\left(r_{1} r_{2}, r_{1} m_{2}+m_{1} r_{2}\right)
$$

This is isomorphic to the ring of all matrices $\left(\begin{array}{ll}r & m \\ 0 & r\end{array}\right)$, where $r \in R$ and $m \in M$ and the usual matrix operations are used.

Corollary 3. A ring $R$ is right (resp. left) $J-M c C o y$ if and only if the trivial extension $T(R, R)$ is a right (resp. left) $J$-McCoy ring.

Let $S$ denote a multiplicatively closed subset of a ring $R$ consisting of central regular elements. Let $R S^{-1}$ be the localization of $R$ at $S$. Then we have:

Theorem 4. For a ring $R$, if $R$ is right (resp. left) J-McCoy, then $R S^{-1}$ is right (resp. left) J-McCoy.

Proof. Suppose that $R$ is right J-McCoy. Let $f(x)=\sum_{i=0}^{n} a_{i} c_{i}^{-1} x^{i}, g(x)=$ $\sum_{j=0}^{m} b_{j} d_{j}^{-1}$

$x^{j}$ be nonzero elements in $\left(R S^{-1}\right)[x]$ such that $f(x) g(x)=0$. Let $a_{i} c_{i}^{-1}=c^{-1} a_{i}^{\prime}$ and $b_{j} d_{j}^{-1}=d^{-1} b_{j}^{\prime}$ with $c, d$ regular elements in $R$. So $f^{\prime}(x) g^{\prime}(x)=0$ such that $f^{\prime}(x)=\sum_{i=0}^{n} a_{i}^{\prime} x^{i}$ and $g^{\prime}(x)=\sum_{j=0}^{m} b_{j}^{\prime} x^{j} \in R[x] \backslash\{0\}$. Since $R$ is right J-McCoy, there exists $r \in R \backslash\{0\}$ such that $a_{i}^{\prime} r \in J(R)$ for each $i$, equivalently we have $1-t a_{i}^{\prime} r$ is left invertible in $R$ for each $t \in R$. So $c^{-1} w^{-1}\left(1-t w^{-1} a_{i} c_{i}^{-1} r c w\right)=c^{-1} w^{-1}-$ 
$t w^{-1} a_{i} c_{i}^{-1} r$ is left invertible in $R S^{-1}$, for each $t w^{-1} \in R S^{-1}$ and so $a_{i} c_{i}^{-1} r c w \in$ $J\left(R S^{-1}\right)$. Thus $R S^{-1}$ is right J-McCoy.

Corollary 4. For a ring $R$, let $R[x]$ be a right (resp. left) J-McCoy ring. Then $R\left[x, x^{-1}\right]$ is a right (resp. left) $J$-McCoy ring.

Proof. Let $\Delta=\left\{1, x, x^{2}, \ldots\right\}$. Then clearly $\Delta$ is multiplicatively closed subset of $R[x]$. Since $R\left[x, x^{-1}\right]=\Delta^{-1} R$, it follows that $R\left[x, x^{-1}\right]$ is right J-McCoy.

A ring $R$ is called right (left) quasi-duo if every maximal right (left) ideal of $R$ is two-sided. It is clear that a ring $R$ is right (left) quasi-duo if and only if $R / J(R)$ is right (left) quasi-duo. Also $R / J(R)$ is a reduced ring in case it is right (left) quasiduo.

Proposition 6. If $R$ is a right (left) quasi-duo ring, then $R$ is right (resp. left) $J-M c C o y$, the converse does not hold in general.

Proof. Since $R$ is right quasi-duo ring, then $R / J(R)$ is reduced by [7] and so $R$ is right $\mathrm{J}-\mathrm{McC}$ oy by Proposition 1. But there exists a right $\mathrm{J}-\mathrm{McC}$ oy ring $R$ which is not right (left) quasi-duo. For instance, take any right primitive domain $R$ that is not division ring (e.g. the free algebra $R=Q<x, y\rangle$ ). Then $R / J(R)=R$ is right $\mathrm{J}$-McCoy, but $R$ is not right quasi-duo by [5].

\section{REFERENCES}

[1] M. Baser, T. K. Kwak, and Y. Lee, "The McCoy condition on skew polynomial rings." Comm. Algebra, pp. 4026-4037, 2009, doi: 10.1080/00927870802545661.

[2] V. Camillo, T. K. Kwak, and Y. Lee, "On a generalization of McCoy rings." J. Korean Math, pp. 959-972, 2013.

[3] M. Habibi, A. Moussavi, and S. Mokhtari, "On skew Armendariz of Laurent series type rings." Comm. Algebra, vol. 40, pp. 3999-4018, 2012, doi: 10.1080/00927872.2011.600746.

[4] M. T. Kosan, "Extention of rings having McCoy condition." Canad. Math. Bull, vol. 52 (2), pp. 267-272, 2009, doi: 10.4153/CMB-2009-029-5.

[5] T. Y. Lam and A. S. Dugas, "Quasi-duo rings and stable range descent." J. Algebra, vol. 195, pp. 243-259, 2005, doi: 10.1016/j.jpaa.2004.08.011.

[6] N. H. McCoy, "Remarks on divisors of zero." American mathematical monthly, vol. 7, no. 4, pp. 286-295, 1942, doi: 10.2307/2268119.

[7] P. P. Nielsen, "Semi-Commutativity and the McCoy condition." J. Algebra, vol. 298, pp. 134-141, 2006, doi: 10.1016/j.jalgebra.2005.10.008.

[8] M. B. Rege and S. Chhawchharia, "Armendariz rings." Proc. Japan Acad. Ser. A Math, vol. 73(A), no. 1, pp. 14-17, 1997, doi: 10.3792/pjaa.73.14.

[9] Z. Ying, J. Chen, and zhen Lei, "Extensions of McCoy rings." Northeast. Math. J., no. 1, pp. 85-94, 2008. 
Authors' addresses

\section{Mohammad Vahdani Mehrabadi}

Department of Mathematics, Islamic Azad University, Central Tehran Branch, 13185/768., Tehran, IRAN.

E-mail address: md_vahdani@yahoo.com

\section{Shervin Sahebi}

Department of Mathematics, Islamic Azad University, Central Tehran Branch, 13185/768., Tehran, IRAN.

E-mail address: sahebi@iauctb.ac.ir

Hamid H. S. Javadi

Department of Mathematics and Computer Science, Shahed University, P. O. Box: 18151-159, Tehran, IRAN.

E-mail address: h.s.javadieshahed.ac.ir 